\title{
On Whitham theory for perturbed integrable equations
}

\author{
A.M. Kamchatnov* \\ Institute of Spectroscopy, Russian Academy of Sciences, \\ Troitsk, Moscow Region, 142190, Russia \\ and \\ Departamento de Física e Centro de Física de Matéria Condensada, \\ Universidade de Lisboa, Av. Prof. Gama Pinto 2, \\ Lisbon 1649-003, Portugal
}

June 26, 2021

\begin{abstract}
Whitham theory of modulations is developed for periodic waves described by nonlinear wave equations integrable by the inverse scattering transform method associated with $2 \times 2$ matrix or second order scalar spectral problems. The theory is illustrated by derivation of the Whitham equations for perturbed Korteweg-de Vries equation and nonlinear Schrödinger equation with linear damping.
\end{abstract}

\section{Introduction}

The theory of perturbations of soliton solutions of integrable equations is well developed and has found many applications to various physical problems (see, e.g. [1]). On the contrary, perturbations of wave trains did not attract much attention, although there are many problems which should be treated in framework of such perturbational approach. As the most evident applications we can mention the theory of dissipationless shock waves in nonlinear media described by the Korteweg-de Vries (KdV) [2], nonlinear Schrödinger (NLS) [3, derivative NLS [4], Kaup-Boussinesq (KB) [5], and many other equations. As other examples one can also mention fluxon trains in long Josephson junctions [6] described by the sine-Gordon equation and soliton trains in Bose-Einstein condensate confined by magnetic traps [7] and described by the NLS equation with external potential. In all these cases unperturbed nonlinear equations have periodic solutions and typical problems can be often reduced to finding slow evolution of parameters of the nonlinear wave due to its initial modulation and small perturbation terms in the governing wave equations.

The general approach to treatment of such problems was suggested long ago by Whitham [8, 9] and it is based on two scales of time - fast oscillations of the wave and slow evolution

*E-mail address: kamch@isan.troitsk.ru 
of its parameters. Then equations for slow evolution of the parameters can be averaged over fast oscillations what leads to the system of the first order partial differential equations called Whitham equations. Whitham discovered that in the case of (unperturbed) KdV equation the arising in this way system can be drastically simplified by introduction of proper parameterization of the periodic solution when Whitham equations take the so-called diagonal form for slowly varying parameters called Riemann invariants. Later it was realized [10] that this property of the $\mathrm{KdV}$ equation is connected with its complete integrability by the inverse scattering transform (IST) method and just this method yields in a natural way the periodic solutions of the KdV equation parameterized by the Riemann invariants. In the fundamental paper [10] Whitham equations were derived for the general case of quasi-periodic solutions of the KdV equation. After that this method was applied to other integrable equations [11, 12, 13] and used for description of dissipationless shock waves in modulationally stable systems [14, 15, 3] and formation of soliton trains at sharp fronts of pulses in modulationally unstable systems (see [16] and references therein).

The Whitham theory approach implies that the process under consideration is considered at asymptotically large periods of time much greater than the period of wave oscillations. However, it is clear that small perturbations neglected in completely integrable approximations can make considerable contribution into long-time evolution. Therefore it is necessary to develop perturbation theory in the Whitham approach and the first step should be development of the method of derivation of perturbed Whitham equations. The method suggested in [17] for perturbed $\mathrm{KdV}$ equation was quite complicated and has not found many applications. Therefore the authors of refs. [18, 19, 20, used the classical Whitham approach for consideration of influence of weak dissipation on long-time evolution of the $\mathrm{KdV}$ wave trains. In a similar way, the Whitham equations for two-phase quasi-periodic solutions of perturbed sine-Gordon equation obtained in [21] did not have effective enough form and were considered in a very special case only.

The aim of this paper is to develop simple and general enough method of obtaining Whitham equations for modulated wave train solutions of perturbed nonlinear equations. The method can be applied to integrable nonlinear wave equations associated, in framework of the IST method, with second order scalar spectral problem or equivalent to it $2 \times 2$ matrix problem (Ablowitz-Kaup-Newell-Segur scheme 22] and its generalizations). The general formulae obtained in the next section are then illustrated by applications to the perturbed $\mathrm{KdV}$ equation (where known results are reproduced) and to the NLS equation with weak linear damping. These examples show that our approach is quite effective and applicable to many nonlinear wave equations of physical importance with various types of perturbations.

\section{Perturbed Whitham equations}

Many physically important equations can be solved, in principle, analytically by the IST method in which they are represented as compatibility conditions of two linear systems with spectral parameter (see, e.g. 23]). We shall confine ourselves to the cases when these two systems can be represented in one of two equivalent forms. The $2 \times 2$ matrix form is given 
by

$$
\begin{aligned}
& \Psi_{x}=\mathbb{U} \Psi, \Psi_{t}=\mathbb{V} \Psi, \\
& \Psi=\left(\begin{array}{c}
\psi_{1} \\
\psi_{2}
\end{array}\right), \quad \mathbb{U}=\left(\begin{array}{cc}
F & G \\
H & -F
\end{array}\right), \quad \mathbb{V}=\left(\begin{array}{cc}
A & B \\
C & -A
\end{array}\right),
\end{aligned}
$$

where the matrix elements depend on as field variables $u_{m}(x, t)$ of the equations under consideration, so the spectral parameter $\lambda$. For example, in the case of the NLS equation,

$$
\mathrm{i} u_{t}+u_{x x} \pm 2|u|^{2} u=0,
$$

these matrix elements correspond to the Zakharov-Shabat spectral problem [24] and are equal to

$$
\begin{aligned}
& F=-\mathrm{i} \lambda, \quad G=\mathrm{i} u, \quad H= \pm \mathrm{i}|u|^{2} ; \\
& A=-2 \mathrm{i} \lambda^{2} \pm \mathrm{i}|u|^{2}, \quad B=2 \mathrm{i} u \lambda-u_{x}, \quad C= \pm 2 \mathrm{i} u^{*} \lambda \pm u_{x}^{*},
\end{aligned}
$$

and there are two field variables, $u(x, t)$ and its complex conjugate $u^{*}(x, t)$. The compatibility condition $\Psi_{x t}=\Psi_{t x}$ of the systems (11) yields at once the evolution equations in general form

$$
\begin{aligned}
& F_{t}-A_{x}+C G-B H=0, \\
& G_{t}-B_{x}+2(B F-A G)=0, \\
& H_{t}-C_{x}+2(A H-C F)=0 .
\end{aligned}
$$

Taking as an example again the NLS equation, it is easy to see that if we substitute (3) into (41) and demand that these equations must fulfil for any value of the spectral parameter $\lambda$, then we find the NLS equation and its complex conjugate,

$$
u_{t}=\mathrm{i} u_{x x} \mp 2 \mathrm{i}|u|^{2} u, \quad u_{t}^{*}=-\mathrm{i} u_{x x}^{*} \pm 2 \mathrm{i}|u|^{2} u^{*} .
$$

In general case (11) we shall write symbolically the equations in terms of the field variables $u_{m}(x, t)$ as

$$
u_{m, t}=K_{m}\left(u_{n}, u_{n, x}, \ldots\right), \quad m, n=1, \ldots, N,
$$

Many physically important nonlinear equations are associated with linear systems (11).

Another common form of linear equations associated with nonlinear integrable equations can be written as

$$
\begin{gathered}
\psi_{x x}=\mathcal{A} \psi \\
\psi_{t}=-\frac{1}{2} \mathcal{B}_{x} \psi+\mathcal{B} \psi_{x},
\end{gathered}
$$

where again the coefficients $\mathcal{A}$ and $\mathcal{B}$ depend on the field variables and the spectral parameter $\lambda$. For well-known example of the KdV equation

$$
u_{t}+6 u u_{x}+u_{x x x}=0
$$

there is only one field variable $u(x, t)$ and

$$
\mathcal{A}=-(u+\lambda), \quad \mathcal{B}=4 \lambda-2 u .
$$

The compatibility condition $\left(\psi_{x x}\right)_{t}=\left(\psi_{t}\right)_{x x}$ of the equations (71), (8) yields

$$
\mathcal{A}_{t}-2 \mathcal{B}_{x} \mathcal{A}-\mathcal{B A}_{x}+\frac{1}{2} \mathcal{B}_{x x x}=0
$$


After substitution of (10) this equation yields the KdV equation (91), and in the general case (17), (8) the resulting evolution equations can be represented again in the form (6) . Besides the $\mathrm{KdV}$ equation, the representation (7), (8) is convenient for treatment of the KB system [25] and some other equations. As was shown in [26], the matrix system (11) can be transformed to scalar form (7), (8) by the formulae

$$
\begin{gathered}
\mathcal{A}=\left(F-G_{x} / 2 G\right)^{2}+G H+\left(F-G_{x} / 2 G\right)_{x}, \\
\mathcal{B}=B / G,
\end{gathered}
$$

so that equations (41) reduce to (11). Thus, these two forms of linear spectral problems are equivalent to each other and both can be used for treatment of the respective nonlinear equations. The scalar form (77),(8) is more convenient for derivation of perturbed Whitham equations and therefore it will be used in what follows.

We assume that unperturbed wave equations (6) have periodic solutions which can be obtained in framework of the IST method in the following way (see, e.g. [23]). We take two basis solutions $\psi^{+}$and $\psi^{-}$of the second order differential equation (7) and construct from them the so-called 'squared basis function'

$$
g=\psi^{+} \psi^{-}
$$

It is easy to show that it satisfies the equation

$$
g_{x x x}-2 \mathcal{A}_{x} g-4 \mathcal{A} g_{x}=0,
$$

which upon multiplication by $g / 2$ can be integrated once to give

$$
\frac{1}{2} g g_{x x}-\frac{1}{4} g_{x}^{2}-\mathcal{A} g^{2}=P(\lambda)
$$

where the integration constant denoted by $P(\lambda)$ can depend on the spectral parameter $\lambda$. In the finite-gap integration method (see, e.g. [27]) it is shown that periodic solutions are distinguished by the condition that $P(\lambda)$ be a polynomial in $\lambda$. Then $g$ as a function of $\lambda$ should also be a polynomial in $\lambda$ with coefficients expressed in terms of $u_{m}(x, t)$, and, hence, $u_{m}(x, t)$ can be found from equations obtained by equating coefficients of $\lambda^{s}$ in Eq. (16) (we suppose here that $\mathcal{A}$ is a rational function of $\lambda$ in accordance with majority of applications). The dependence of $g$, and hence of its coefficients, on time $t$ is obtained from the equation

$$
g_{t}=\mathcal{B} g_{x}-\mathcal{B}_{x} g
$$

which follows from Eqs. (8) and (14). As a result, $u_{m}(x, t)$ are expressed as (quasi)periodic functions of $x$ and $t$ parameterized by the zeroes $\lambda_{i}$ of the polynomial $P(\lambda)=\prod_{i=1}^{M}\left(\lambda-\lambda_{i}\right)$,

$$
u_{m}(x, t)=u_{m}\left(x, t ; \lambda_{1}, \ldots, \lambda_{M}\right), \quad m=1, \ldots, N .
$$

In strictly periodic solutions the parameters $\lambda_{i}$ are constant by definition. But we can slightly modulate this periodic wave by making $\lambda_{i}$ slow functions of $x$ and $t$ which change 
little in one wavelength and one period. Whitham showed [8, 9] (for particular case of onephase periodic solution of the KdV equation when this solution is parameterized by three zeroes $\left.\lambda_{1}, \lambda_{2}, \lambda_{3}\right)$ that substitution of

$$
u_{m}(x, t)=u_{m}\left(x, t ; \lambda_{1}(x, t), \ldots, \lambda_{M}(x, t)\right), \quad m=1, \ldots, N,
$$

into conservation laws of Eqs. (6) followed by averaging of these conservation laws over fast oscillations of the wave yields for slow variables $\lambda_{i}(x, t)$ the so-called Whitham modulation equations

$$
\frac{\partial \lambda_{i}}{\partial t}+v_{i}\left(\lambda_{j}\right) \frac{\partial \lambda_{i}}{\partial x}=0, \quad i, j=1, \ldots, M
$$

which govern slow evolution of modulated periodic solutions of unperturbed equations (6). This approach is well developed and finds a number of applications (see, e.g. 23]).

Now, we want to take into account perturbations in the wave equations (6),

$$
u_{m, t}=K_{m}\left(u_{n}, u_{n, x}, \ldots\right)+R_{m}\left(x, t, u_{n}, u_{n, x}, \ldots\right), \quad m, n=1, \ldots, N,
$$

where the perturbation terms $R_{m}$ can be slow functions of $x$ and $t$ and can also depend on the field variables $u_{n}$ and their space derivatives. The perturbation terms can have any nature, e.g., they can arise due to external potentials or small physical effects neglected in derivation of unperturbed equations (6). Obviously, the functions (18) are not the solutions of the perturbed equations (21) anymore, but again, as in the case of slow modulation, we can describe effects of perturbations as slow change of the parameters $\lambda_{i}, i=1, \ldots, M$. Then after averaging over fast oscillations of the wave we have to obtain additional terms in the Whitham equations (20) which represent effects of perturbations. Our aim is to derive such generalization of the Whitham equations.

As was indicated by Whitham [8, 9], the most convenient way of obtaining the modulation equations is to average the conservation laws. Completely integrable equations have an infinite sequence of conservation laws and averaging of their generating function yields for periodic solutions of equations (6) associated with the spectral problems (11) or (17), (8) the modulation Whitham equations directly in diagonal Riemann form [10]. Therefore the starting point of our analysis should be the formula for generating function of conservation laws for equations (11) described by the IST scheme (17), (8). As was mentioned above, we suppose that $\mathcal{A}$ is a rational function of $\lambda$. Then it can be chosen so that

$$
\left.\mathcal{A}\right|_{\lambda \rightarrow \infty} \longrightarrow \sigma \lambda^{r}
$$

where $\sigma= \pm 1$ and $r$ is an integer number. With such a normalization of $\mathcal{A}$, we can rewrite Eq. (16) in the form

$$
\frac{1}{2} g g_{x x}-\frac{1}{4} g_{x}^{2}-\mathcal{A} g^{2}=-\sigma P(\lambda)
$$

where $P$ and $g$ are polynomials in $\lambda$,

$$
P=\prod_{i=1}^{M}\left(\lambda-\lambda_{i}\right), \quad g=\prod_{i=1}^{\operatorname{deg} g}\left(\lambda-\mu_{i}(x, t)\right),
$$


such that their degrees are connected by the relation $M=r+2 \operatorname{deg} g$. If we introduce new function

$$
\tilde{g}=\frac{\lambda^{r / 2}}{\sqrt{P(\lambda)}} g
$$

then the identity (23) takes the form

$$
\frac{1}{2 \lambda^{r}} \tilde{g} \tilde{g}_{x x}-\frac{1}{4 \lambda^{r}} \tilde{g}_{x}^{2}-\tilde{\mathcal{A}} \tilde{g}^{2}=-\sigma,
$$

where

$$
\tilde{\mathcal{A}}=\mathcal{A} / \lambda^{r}
$$

and

$$
\left.\tilde{g}\right|_{\lambda \rightarrow \infty}=1 .
$$

Thus, we can expand $\tilde{g}$ in inverse powers of $\lambda$,

$$
\tilde{g}=\sum_{n=0}^{\infty} \frac{g_{n}}{\lambda^{n}}, \quad g_{0}=1,
$$

and the coefficients $g_{n}$ can be calculated in recurrent way by substitution of (29) into (26). The examples of this procedure will be given below.

Now, from (17) and (25) we find that $\tilde{g}$ satisfies the relation

$$
\left(\frac{1}{\tilde{g}}\right)_{t}=\left(\frac{\mathcal{B}}{\tilde{g}}\right)_{x}
$$

which can be considered as a generating function of conservation laws. Indeed, the coefficients of the expansion

$$
\frac{1}{\tilde{g}}=\sum_{n=0}^{\infty} \frac{g_{-n}}{\lambda^{n}}, \quad g_{0}=1,
$$

can be expressed in terms of the already known coefficients $g_{n}$ with the use of trivial identity $\tilde{g} \cdot(1 / \tilde{g})=1$, and then these coefficients $g_{-n}$ serve as the densities of the conservation laws and the coefficients of similar expansion of $\mathcal{B} / \tilde{g}$ in inverse powers of $\lambda$ serve as the corresponding fluxes,

$$
\left(g_{-n}\right)_{t}=\left[(\mathcal{B} / \tilde{g})_{-n}\right]_{x},
$$

where $(\mathcal{B} / \tilde{g})_{-n}$ denotes the coefficients in the expansion

$$
\frac{\mathcal{B}}{\tilde{g}}=\sum_{n=0}^{\infty} \frac{(\mathcal{B} / \tilde{g})_{-n}}{\lambda^{n}}
$$

The functions $\tilde{g}$ and $1 / \tilde{g}$ satisfy important identities

$$
\frac{\widehat{\delta}}{\delta u_{m}}\left(\frac{1}{\tilde{g}}\right)=\frac{\sigma}{2 \lambda^{r}} \sum_{l=0}^{A_{m}}(-1)^{l} \frac{\partial^{l}}{\partial x^{l}}\left(\frac{\partial \mathcal{A}}{\partial u_{m}^{(l)}} \tilde{g}\right), \quad m=1, \ldots, N,
$$


derived in the Appendix. Here $\widehat{\delta} / \delta u_{m}$ denotes the "Euler derivative"

$$
\frac{\widehat{\delta}}{\delta u_{m}}=\frac{\partial}{\partial u_{m}}-\frac{\partial}{\partial x} \frac{\partial}{\partial u_{m, x}}+\frac{\partial^{2}}{\partial x^{2}} \frac{\partial}{\partial u_{m, x x}}-\ldots+(-1)^{N_{m n}} \frac{\partial^{N_{m n}}}{\partial x^{N_{m n}}} \frac{\partial}{\partial u_{m}^{\left(N_{m n}\right)}},
$$

when it is applied to $g_{-n}, N_{m n}$ is the order of the highest derivative $u_{m}^{\left(N_{m n}\right)}$ in $g_{-n}$, and $A_{m}$ is the order of the highest derivative $u_{m}^{\left(A_{m}\right)}$ in $\mathcal{A}$.

Now let us turn to perturbed equations (21) and calculate the correction to the conservation law (32) with the density

$$
g_{-n}=g_{-n}\left(u_{m}, u_{m, x}, \ldots, u_{m}^{\left(N_{m n}\right)}\right) .
$$

Evidently, we have

$$
\begin{aligned}
\left(g_{-n}\right)_{t} & =\sum_{m, k} g_{-n, m k} \partial_{t} u_{m}^{(k)}=\sum_{m, k} g_{-n, m k}\left(\partial_{x}\right)^{k} \partial_{t} u_{m} \\
& =\sum_{m, k} g_{-n, m k}\left(\partial_{x}\right)^{k} K_{m}+\sum_{m, k} g_{-n, m k}\left(\partial_{x}\right)^{k} R_{m}
\end{aligned}
$$

where $g_{-n, m k}$ denotes the derivative of $g_{-n}$ with respect to $u_{m}^{(k)}$. The terms with $K_{m}$ must collect into divergence of the flux, $\partial_{x}\left[(\mathcal{B} / \tilde{g})_{-n}\right]$, so that we obtain

$$
\left(g_{-n}\right)_{t}=\left[(\mathcal{B} / \tilde{g})_{-n}\right]_{x}+\sum_{m, k} g_{-n, m k}\left(\partial_{x}\right)^{k} R_{m}
$$

In what follows we shall average these conservation laws over some interval $\Lambda$ of $x$ which is supposed to be much greater than the wavelength $L$ and much less than the characteristic interval along which the parameters of the wave change considerably. Therefore we can transform the terms with $R_{m}$ by "integration by parts" and neglect the contribution of boundaries because in the averaged form it is divided by $\Lambda$ and goes to zero in asymptotic limit $\Lambda \gg L$ of very slow modulation. As a result of this transformation we obtain the conservation law in the following equivalent form:

$$
\left(g_{-n}\right)_{t}=\left[\left(\frac{\mathcal{B}}{\tilde{g}}\right)_{-n}\right]_{x}+\sum_{m}\left(\frac{\widehat{\delta}}{\delta u_{m}} g_{-n}\right) R_{m}
$$

After dividing this formula by $\lambda^{n}$ and summation over $n$ we arrive at the generating function of perturbed conservation laws

$$
\left(\frac{1}{\tilde{g}}\right)_{t}=\left(\frac{\mathcal{B}}{\tilde{g}}\right)_{x}+\sum_{m}\left(\frac{\widehat{\delta}}{\delta u_{m}} \frac{1}{\tilde{g}}\right) R_{m}
$$

For further transformation we apply the identity (34) and get

$$
\left(\frac{1}{\tilde{g}}\right)_{t}-\left(\frac{\mathcal{B}}{\tilde{g}}\right)_{x}=\frac{\sigma}{2 \lambda^{r}} \sum_{m, l}(-1)^{l} \frac{\partial^{l}}{\partial x^{l}}\left(\frac{\partial \mathcal{A}}{\partial u_{m}^{(l)}} \tilde{g}\right) R_{m}
$$

Here we again can "integrate by parts" and neglect the boundary terms to obtain

$$
\left(\frac{1}{\tilde{g}}\right)_{t}-\left(\frac{\mathcal{B}}{\tilde{g}}\right)_{x}=\frac{\sigma}{2 \lambda^{r}} \sum_{m, l} \frac{\partial \mathcal{A}}{\partial u_{m}^{(l)}} \frac{\partial^{l} R_{m}}{\partial x^{l}} \tilde{g}
$$


To average this expression, it is convenient to return to the squared basis function $g$ with the help of Eq. (17),

$$
\left(\frac{\sqrt{P(\lambda)}}{g}\right)_{t}-\left(\sqrt{P(\lambda)} \frac{\mathcal{B}}{g}\right)_{x}=\frac{\sigma}{2} \sum_{m, l} \frac{\partial \mathcal{A}}{\partial u_{m}^{(l)}} \frac{\partial^{l} R_{m}}{\partial x^{l}} \frac{g}{\sqrt{P(\lambda)}} .
$$

Here the zeroes $\lambda_{i}$ of the polynomial $P(\lambda)$ are slow functions of $x$ and $t$ which have to be differentiated with respect to $x$ and $t$ in the left hand side of Eq. (40). Then we obtain the terms with

$$
\frac{1}{\sqrt{\lambda-\lambda_{i}}} \frac{\partial \lambda_{i}}{\partial t} \text { and } \frac{1}{\sqrt{\lambda-\lambda_{i}}} \frac{\partial \lambda_{i}}{\partial x}
$$

which are singular at $\lambda \rightarrow \lambda_{i}$, and in the right hand side of Eq. (40) we already have terms with the same singular behaviour. Since the perturbed conservation laws (40) after averaging should be true at any $\lambda$ including $\lambda_{i}$, we obtain

$$
\frac{\partial \lambda_{i}}{\partial t}+v_{i} \frac{\partial \lambda_{i}}{\partial x}=\frac{\sigma}{2\langle 1 / g\rangle \prod_{j \neq i}\left(\lambda_{i}-\lambda_{j}\right)} \sum_{m=1}^{N} \sum_{l=0}^{A_{m}}\left\langle\frac{\partial \mathcal{A}}{\partial u_{m}^{(l)}} \frac{\partial^{l} R_{m}}{\partial x^{l}} g\right\rangle, \quad i=1, \ldots, M
$$

where

$$
v_{i}=-\frac{\langle\mathcal{B} / g\rangle}{\langle 1 / g\rangle}, \quad i=1, \ldots, M
$$

angle brackets denote averaging over proper interval of $x$, and everywhere $\lambda$ is put equal to $\lambda_{i}$. Formula (42) gives well-known expressions for characteristic velocities in unperturbed Whitham equations. The right hand side of (41) describes the effects of perturbations. Equations (41) represent the result we wanted to obtain. The averaged values of the expressions in angle brackets can be evaluated with the use of formulae for periodic solutions of particular integrable equations under consideration (see, e.g., [10, 28]).

Let us illustrate the obtained expressions for perturbed Whitham equations with two examples.

\section{Examples}

We shall consider here simple examples of application of general formulae (41), (42) to onephase periodic solutions of the KdV and NLS equations.

\subsection{Korteweg-de Vries equation}

In the case of the $\mathrm{KdV}$ equation

$$
u_{t}+6 u u_{x}+u_{x x x}=R
$$

we have expressions (10) for $\mathcal{A}$ and $\mathcal{B}$. From (26) we find the expansion

$$
\tilde{g}=1-\frac{u / 2}{\lambda}+\frac{1}{\lambda^{2}}\left(\frac{3}{8} u^{2}+\frac{1}{8} u_{x x}\right)-\frac{1}{\lambda^{3}}\left(\frac{5}{16} u^{3}+\frac{5}{32} u_{x}^{2}+\frac{5}{16} u u_{x x}+\frac{1}{32} u^{(I V)}\right)+\ldots
$$


hence

$$
\frac{1}{\tilde{g}}=1+\frac{u / 2}{\lambda}-\frac{1}{\lambda^{2}}\left(\frac{1}{8} u^{2}+\frac{1}{8} u_{x x}\right)+\frac{1}{\lambda^{3}}\left(\frac{1}{16} u^{3}+\frac{5}{32} u_{x}^{2}+\frac{3}{16} u u_{x x}+\frac{1}{32} u^{(I V)}\right)+\ldots,
$$

and it is easy to check that these first terms of the expansions satisfy the identity (34), i.e.

$$
\frac{\widehat{\delta}}{\delta u} \frac{1}{\tilde{g}}=\frac{\tilde{g}}{2 \lambda} .
$$

The conservation laws following from Eq. (32) have well-known form (see, e.g. 23]).

The periodic solution ("cnoidal wave") of unperturbed KdV equation has the form (see, e.g. [23])

$$
u(x, t)=\lambda_{3}-\lambda_{1}-\lambda_{2}-2\left(\lambda_{3}-\lambda_{2}\right) \operatorname{sn}^{2}\left(\sqrt{\lambda_{3}-\lambda_{1}}(x-V t), m\right),
$$

where

$$
V=-2\left(\lambda_{1}+\lambda_{2}+\lambda_{3}\right), \quad m=\frac{\lambda_{3}-\lambda_{2}}{\lambda_{3}-\lambda_{1}},
$$

and the parameters $\lambda_{1} \leq \lambda_{2} \leq \lambda_{3}$ are the zeroes of the third degree polynomial

$$
P(\lambda)=\prod_{i=1}^{3}\left(\lambda-\lambda_{i}\right)=\lambda^{3}-s_{1} \lambda^{2}+s_{2} \lambda-s_{3}
$$

defining the solution according to Eq. (16). Then

$$
g(x, t)=\lambda-\mu(x, t),
$$

where $\mu(x, t)$ is connected with $u(x, t)$ by the relation

$$
u=2 \mu-\left(\lambda_{1}+\lambda_{2}+\lambda_{3}\right)=2 \mu-s_{1}
$$

and depends only on the phase

$$
\theta=x-V t, \quad \mu_{\theta}=2 \sqrt{-P(\mu)}, \quad \lambda_{2} \leq \mu \leq \lambda_{3} .
$$

The wavelength of the solution (47) is given by the expression

$$
L=\frac{1}{2} \oint \frac{\mathrm{d} \mu}{\sqrt{-P(\mu)}}=\int_{\lambda_{2}}^{\lambda_{3}} \frac{\mathrm{d} \mu}{\sqrt{-P(\mu)}}=\frac{2 \mathrm{~K}(m)}{\sqrt{\lambda_{3}-\lambda_{1}}},
$$

$\mathrm{K}(m)$ being the complete elliptic integral of the first kind. In this one-phase case we can average over wavelength and the Whitham velocities are easily expressed in terms of $L$ by means of the formula

$$
\left\langle\frac{1}{g}\right\rangle_{\lambda=\lambda_{i}}=\left\langle\frac{1}{\lambda_{i}-\mu}\right\rangle=\frac{1}{L} \int_{0}^{L} \frac{\mathrm{d} x}{\lambda_{i}-\mu}=\frac{1}{2 L} \oint \frac{\mathrm{d} \mu}{\left(\lambda_{i}-\mu\right) \sqrt{-P(\mu)}}=-\frac{2}{L} \frac{\partial L}{\partial \lambda_{i}},
$$

so that we obtain

$$
v_{i}=-2 s_{1}+\frac{2 L}{\partial L / \partial \lambda_{l}}=\left(1-\frac{L}{\partial_{i} L} \partial_{i}\right) V, \quad \partial_{i} \equiv \frac{\partial}{\partial \lambda_{i}}, \quad i=1,2,3 .
$$


Substitution of (53) into this expression yields the well-known formulae obtained by Whitham [8. 9. In the $\mathrm{KdV}$ equation case the sum in the right hand side of Eq. (41) reduces to a single term with $\partial \mathcal{A} / \partial u=-1$. Taking into account that $\sigma=-1, g=\lambda_{i}-\mu=\left(2 \lambda_{i}-s_{1}-u\right) / 2$, we arrive at

$$
\frac{\partial \lambda_{i}}{\partial t}+v_{i} \frac{\partial \lambda_{i}}{\partial x}=\frac{L}{\partial L / \partial \lambda_{i}} \frac{\left\langle\left(2 \lambda_{i}-s_{1}-u\right) R\right\rangle}{4 \prod_{j \neq i}\left(\lambda_{i}-\lambda_{j}\right)}, \quad i=1,2,3 .
$$

This is a compact expression for perturbed Whitham equations obtained in [20].

Further calculation can be done after specification of the perturbation $R$. For example, if we take

$$
R=\varepsilon u_{x x}
$$

( $\varepsilon$ is a small numeric parameter) what corresponds to taking into account small viscosity in hydrodynamic applications of the KdV equation, then we have

$$
u_{x x}=2 \mu_{x x}=-2 \frac{\mathrm{d} P}{\mathrm{~d} \mu}, \quad 2 \lambda_{i}-s_{1}-u=2\left(\lambda_{i}-\mu\right),
$$

so that

$$
\begin{aligned}
\left\langle\left(2 \lambda_{i}-s_{1}-u\right) \varepsilon u_{x x}\right\rangle & =-\frac{4 \varepsilon}{L} \oint \frac{\lambda_{i}-\mu}{\sqrt{-P(\mu)}} \frac{\mathrm{d} P}{\mathrm{~d} \mu} \mathrm{d} \mu=\frac{8 \varepsilon}{L} \oint \sqrt{-P(\mu)} \mathrm{d} \mu \\
& =\frac{16 \varepsilon}{L} \int_{\lambda_{2}}^{\lambda_{3}} \sqrt{-P(\mu)} \mathrm{d} \mu,
\end{aligned}
$$

and standard calculation of this elliptic integral yields

$$
\begin{array}{r}
\left\langle\left(2 \lambda_{i}-s_{1}-u\right) \varepsilon u_{x x}\right\rangle=\frac{32 \varepsilon}{15} \frac{\left(\lambda_{3}-\lambda_{1}\right)^{3}}{\mathrm{~K}(m)}\left[\left(1-m+m^{2}\right) \mathrm{E}(m)\right. \\
-(1-m)(1-m / 2) \mathrm{K}(m)],
\end{array}
$$

$\mathrm{E}(m)$ being the complete elliptic integral of the second kind. Substitution of (58) into (56) gives the perturbed Whitham equations for the KdV-Burgers equation found first in [18, 19]. Thus, the general formula (41) reproduces quite easily the already known results for the perturbed KdV equation.

\subsection{Nonlinear Schrödinger equation}

As the second example, let us consider the focusing NLS equation with linear damping

$$
\mathrm{i} u_{t}+u_{x x}+2|u|^{2} u=-\mathrm{i} \varepsilon u .
$$

(Similar results for the defocusing NLS equation will be presented in the end of this Section.) The unperturbed equation is usually associated in framework of the IST method with the $2 \times 2$ Zakharov-Shabat spectral problem [24. However, with the use of Eqs. (12), (13) it can be transformed into scalar form (178) with (see [29, 26])

$$
\mathcal{A}=-\left(\lambda-\frac{\mathrm{i} u_{x}}{2 u}\right)^{2}-|u|^{2}-\left(\frac{u_{x}}{2 u}\right)_{x}, \quad \mathcal{B}=2 \lambda+\frac{\mathrm{i} u_{x}}{u} .
$$

Expansions of $\tilde{g}$ and $1 / \tilde{g}$ are given by

$$
\tilde{g}=1+\frac{1}{\lambda} \frac{\mathrm{i} u_{x}}{2 u}-\frac{1}{\lambda^{2}}\left(\frac{|u|^{2}}{2}+\frac{u_{x x}}{4 u}\right)-\frac{1}{\lambda^{3}}\left(\frac{\mathrm{i} u_{x x x}}{8 u}-\frac{3 \mathrm{i} u_{x} u^{*}}{4}\right)+\ldots,
$$




$$
\begin{array}{r}
\frac{1}{\tilde{g}}=1-\frac{1}{\lambda} \frac{\mathrm{i} u_{x}}{2 u}+\frac{1}{\lambda^{2}}\left(\frac{|u|^{2}}{2}+\frac{u_{x x}}{4 u}-\frac{u_{x}^{2}}{4 u^{2}}\right) \\
+\frac{1}{\lambda^{3}}\left(\frac{\mathrm{i} u_{x x x}}{8 u}+\frac{\mathrm{i} u_{x} u^{*}}{4}-\frac{\mathrm{i} u_{x} u_{x x}}{4 u^{2}}+\frac{\mathrm{i} u_{x}^{3}}{8 u^{3}}\right)+\ldots,
\end{array}
$$

and it is easy to check that they satisfy the identities

$$
\begin{gathered}
\frac{\widehat{\delta}}{\delta u} \frac{1}{\tilde{g}}=-\frac{1}{2 \lambda^{2}}\left[\frac{\partial \mathcal{A}}{\partial u} \tilde{g}-\frac{\partial}{\partial x}\left(\frac{\partial \mathcal{A}}{\partial u_{x}} \tilde{g}\right)+\frac{\partial^{2}}{\partial x^{2}}\left(\frac{\partial \mathcal{A}}{\partial u_{x x}} \tilde{g}\right)\right], \\
\frac{\widehat{\delta}}{\delta u^{*}} \frac{1}{\tilde{g}}=\frac{u}{2 \lambda^{2}} \tilde{g} .
\end{gathered}
$$

The generating function (30) yields the conservation laws in unusual form

$$
\begin{gathered}
\left(\frac{\mathrm{i} u_{x}}{u}\right)_{t}=-\left(2|u|^{2}+\frac{u_{x x}}{u}\right)_{x}, \\
\left(\mathrm{i}|u|^{2}+\frac{\mathrm{i} u_{x x}}{2 u}-\frac{\mathrm{i} u_{x}^{2}}{2 u^{2}}\right)_{t}=\left(\frac{u_{x} u_{x x}}{2 u^{2}}-\frac{u_{x x x}}{2 u}-2 u_{x} u^{*}\right)_{x}, \ldots
\end{gathered}
$$

The one-phase periodic solution is parameterized by two pairs of complex conjugated zeroes $\lambda_{i}$ of the polynomial (see [23, 30])

$$
\begin{gathered}
P(\lambda)=\prod_{i=1}^{4}\left(\lambda-\lambda_{i}\right)=\lambda^{4}-s_{1} \lambda^{3}+s_{2} \lambda^{2}-s_{3} \lambda+s_{4}, \\
\lambda_{1}=\alpha+\mathrm{i} \gamma, \quad \lambda_{2}=\beta+\mathrm{i} \delta, \quad \lambda_{3}=\alpha-\mathrm{i} \gamma, \quad \lambda_{4}=\beta-\mathrm{i} \delta,
\end{gathered}
$$

so that the intensity of the wave is given by

$$
\begin{aligned}
& \nu=|u(x, t)|^{2}=\nu_{3}-\left(\nu_{3}-\nu_{2}\right) \operatorname{sn}^{2}\left(\sqrt{\nu_{3}-\nu_{1}} \theta, m\right) \\
& =(\gamma+\delta)^{2}-4 \gamma \delta \operatorname{sn}^{2}\left(\sqrt{(\alpha-\beta)^{2}+(\gamma+\delta)^{2}} \theta, m\right),
\end{aligned}
$$

where

$$
m=\frac{\nu_{3}-\nu_{2}}{\nu_{3}-\nu_{1}}=\frac{\left(\lambda_{1}-\lambda_{3}\right)\left(\lambda_{2}-\lambda_{4}\right)}{\left(\lambda_{1}-\lambda_{4}\right)\left(\lambda_{2}-\lambda_{3}\right)}=\frac{4 \gamma \delta}{(\alpha-\beta)^{2}+(\gamma+\delta)^{2}},
$$

$\nu_{1}, \nu_{2}, \nu_{3}$ are the zeroes of the resolvent $\mathcal{R}(\nu)$ of the polynomial $P(\lambda)$,

$$
\begin{gathered}
\nu_{1}=-\frac{1}{4}\left(\lambda_{1}-\lambda_{2}+\lambda_{3}-\lambda_{4}\right)^{2}=-(\alpha-\beta)^{2}, \\
\nu_{2}=-\frac{1}{4}\left(\lambda_{1}-\lambda_{2}-\lambda_{3}+\lambda_{4}\right)^{2}=(\gamma-\delta)^{2}, \\
\nu_{3}=-\frac{1}{4}\left(\lambda_{1}+\lambda_{2}-\lambda_{3}-\lambda_{4}\right)^{2}=(\gamma+\delta)^{2},
\end{gathered}
$$


and $\nu$ satisfies the equation

$$
\nu_{\theta}=2 \sqrt{-\mathcal{R}(\nu)}, \quad \mathcal{R}(\nu)=\prod_{i=1}^{3}\left(\nu-\nu_{i}\right), \quad \nu_{2} \leq \nu \leq \nu_{3},
$$

$\theta$ being the phase of the wave

$$
\theta=x-V t, \quad V=-s_{1}=-\sum_{i=1}^{4} \lambda_{i}
$$

In this case there are two fields $u$ and $u^{*}$, and the corresponding perturbations are equal to

$$
R=-\varepsilon u, \quad R^{*}=-\varepsilon u^{*},
$$

where $\varepsilon$ is a small constant parameter. Easy calculation gives

$$
\frac{\partial \mathcal{A}}{\partial u} R+\frac{\partial \mathcal{A}}{\partial u_{x}} \frac{\partial R}{\partial x}+\frac{\partial \mathcal{A}}{\partial u_{x x}} \frac{\partial^{2} R}{\partial x^{2}}+\frac{\partial \mathcal{A}}{\partial u^{*}} R^{*}=2 \varepsilon|u|^{2}=2 \varepsilon \nu,
$$

so that we have to average the expression $2 \varepsilon \nu g$. Now, $g(x, t)=\lambda-\mu(x, t)$, where $\mu$ satisfies the equation

$$
\mu_{\theta}=2 \sqrt{-P(\mu)}
$$

and can be expressed as a function of $\nu$ (see [16, 23, 30]),

$$
\begin{array}{r}
\mu(\nu)=\frac{s}{4}-\frac{q+\mathrm{i} \sqrt{-\mathcal{R}(\nu)}}{2 \nu}, \quad \nu_{2} \leq \nu \leq \nu_{3}, \\
s=s_{1}, \quad q=\sqrt{-\nu_{1} \nu_{2} \nu_{3}} .
\end{array}
$$

Thus, we obtain

$$
\begin{array}{r}
\langle 2 \varepsilon \nu g\rangle=\frac{2 \varepsilon}{L} \int_{0}^{L} \nu\left(\lambda_{i}-\mu\right) \mathrm{d} x=\frac{\varepsilon}{L} \oint \nu\left(\lambda_{i}-\frac{s}{4}+\frac{q+\mathrm{i} \sqrt{-\mathcal{R}(\nu)}}{2 \nu}\right) \frac{\mathrm{d} \nu}{\sqrt{-\mathcal{R}(\nu)}} \\
=\frac{2 \varepsilon}{L} \int_{\nu_{2}}^{\nu_{3}} \frac{\left(\lambda_{i}-s / 4\right) \nu+q / 2}{\sqrt{\left(\nu_{3}-\nu\right)\left(\nu-\nu_{2}\right)\left(\nu-\nu_{1}\right)}} \mathrm{d} \nu
\end{array}
$$

where

$$
L=\frac{1}{2} \oint \frac{\mathrm{d} \mu}{\sqrt{-P(\mu)}}=\int_{\nu_{2}}^{\nu_{3}} \frac{\mathrm{d} \nu}{\sqrt{-\mathcal{R}(\nu)}}=\frac{2 \mathrm{~K}(m)}{\sqrt{\nu_{3}-\nu_{1}}}=\frac{2 \mathrm{~K}(m)}{\sqrt{\left(\lambda_{1}-\lambda_{4}\right)\left(\lambda_{3}-\lambda_{2}\right)}} .
$$

The integral in (177) reduces to standard elliptic integrals,

$$
\langle 2 \varepsilon \nu g\rangle=\frac{2 \varepsilon}{L \sqrt{\nu_{3}-\nu_{1}}}\left\{\left[\left(2 \lambda_{i}-s / 2\right) \nu_{1}+q\right] \mathrm{K}(m)+\left(2 \lambda_{i}-s / 2\right)\left(\nu_{3}-\nu_{1}\right) \mathrm{E}(m)\right\} .
$$

The averaged values $\langle 1 / g\rangle$ and $\langle\mathcal{B} / g\rangle$ are calculated as in the previous cases of the $\mathrm{KdV}$ equation. As a result, we arrive at the perturbed Whitham equations in the form

$$
\frac{\partial \lambda_{i}}{\partial t}+v_{i} \frac{\partial \lambda_{i}}{\partial x}=-\varepsilon \rho_{i}, \quad i=1,2,3,4,
$$


where Whitham velocities are given by the expression

$$
v_{i}=-\frac{\langle\mathcal{B} / g\rangle}{\langle 1 / g\rangle}=-s_{1}+\frac{L}{\partial L / \partial \lambda_{l}}=\left(1-\frac{L}{\partial_{i} L} \partial_{i}\right) V, \quad i=1,2,3,4,
$$

which reduces after substitution of (78) to well-known formulae [12, 13, and functions $\rho_{i}$ in the right hand side of Eq. (80) are equal to

$$
\begin{gathered}
\rho_{1}=-\frac{\left[\left(2 \lambda_{1}-s / 2\right) \nu_{1}+q\right] \mathrm{K}(m)+\left(2 \lambda_{1}-s / 2\right)\left(\nu_{3}-\nu_{1}\right) \mathrm{E}(m)}{\left(\lambda_{1}-\lambda_{4}\right)\left[\left(\lambda_{1}-\lambda_{2}\right) \mathrm{K}(m)+\left(\lambda_{2}-\lambda_{3}\right) \mathrm{E}(m)\right]}, \\
\rho_{2}=\frac{\left[\left(2 \lambda_{2}-s / 2\right) \nu_{1}+q\right] \mathrm{K}(m)+\left(2 \lambda_{2}-s / 2\right)\left(\nu_{3}-\nu_{1}\right) \mathrm{E}(m)}{\left(\lambda_{2}-\lambda_{3}\right)\left[\left(\lambda_{1}-\lambda_{2}\right) \mathrm{K}(m)-\left(\lambda_{1}-\lambda_{4}\right) \mathrm{E}(m)\right]}, \\
\rho_{3}=\frac{\left[\left(2 \lambda_{3}-s / 2\right) \nu_{1}+q\right] \mathrm{K}(m)+\left(2 \lambda_{3}-s / 2\right)\left(\nu_{3}-\nu_{1}\right) \mathrm{E}(m)}{\left(\lambda_{2}-\lambda_{3}\right)\left[\left(\lambda_{3}-\lambda_{4}\right) \mathrm{K}(m)-\left(\lambda_{1}-\lambda_{4}\right) \mathrm{E}(m)\right]}, \\
\rho_{4}=-\frac{\left[\left(2 \lambda_{4}-s / 2\right) \nu_{1}+q\right] \mathrm{K}(m)+\left(2 \lambda_{4}-s / 2\right)\left(\nu_{3}-\nu_{1}\right) \mathrm{E}(m)}{\left(\lambda_{1}-\lambda_{4}\right)\left[\left(\lambda_{3}-\lambda_{4}\right) \mathrm{K}(m)+\left(\lambda_{2}-\lambda_{3}\right) \mathrm{E}(m)\right]} .
\end{gathered}
$$

Equations (81) determine evolution of the Riemann invariants $\lambda_{i}, i=1,2,3,4$, due to combined action of modulation and perturbation effects.

For perturbed defocusing NLS equation

$$
\mathrm{i} u_{t}+u_{x x}+2|u|^{2} u=-\mathrm{i} \varepsilon u
$$

calculations are actually the same, so we present here only the final results. The intensity of periodic wave is given by the formula

$$
\begin{array}{r}
\nu=|u(x, t)|^{2}=\bar{\nu}_{1}+\left(\bar{\nu}_{2}-\bar{\nu}_{1}\right) \operatorname{sn}^{2}\left(\sqrt{\bar{\nu}_{2}-\bar{\nu}_{1}} \theta, m\right) \\
=\frac{1}{4}\left(\lambda_{1}-\lambda_{2}-\lambda_{3}+\lambda_{4}\right)^{2}+\left(\lambda_{2}-\lambda_{1}\right)\left(\lambda_{4}-\lambda_{3}\right) \operatorname{sn}^{2}\left(\sqrt{\left(\lambda_{3}-\lambda_{1}\right)\left(\lambda_{4}-\lambda_{2}\right)} \theta, m\right)
\end{array}
$$

where the zeroes $\lambda_{i}, i=1,2,3,4$, of the fourth degree polynomial $P(\lambda)$ are real Riemann invariants, $\lambda_{1} \leq \lambda_{2} \leq \lambda_{3} \leq \lambda_{4}$, connected with $\bar{\nu}_{i}, i=1,2,3$, by the formulae

$$
\begin{aligned}
\bar{\nu}_{1}=\frac{1}{4}\left(\lambda_{1}-\lambda_{2}-\lambda_{3}+\lambda_{4}\right)^{2}, \quad \bar{\nu}_{2} & =\frac{1}{4}\left(\lambda_{1}-\lambda_{2}+\lambda_{3}-\lambda_{4}\right)^{2}, \\
\bar{\nu}_{3} & =\frac{1}{4}\left(\lambda_{1}+\lambda_{2}-\lambda_{3}-\lambda_{4}\right)^{2},
\end{aligned}
$$

and

$$
\begin{gathered}
m=\frac{\bar{\nu}_{2}-\bar{\nu}_{1}}{\bar{\nu}_{3}-\bar{\nu}_{1}}=\frac{\left(\lambda_{2}-\lambda_{1}\right)\left(\lambda_{4}-\lambda_{3}\right)}{\left(\lambda_{3}-\lambda_{1}\right)\left(\lambda_{4}-\lambda_{2}\right)}, \\
\theta=x-V t, \quad V=-s_{1}=-\sum_{i=1}^{4} \lambda_{i} .
\end{gathered}
$$

The wavelength is equal to

$$
L=\frac{2 \mathrm{~K}(m)}{\sqrt{\bar{\nu}_{3}-\bar{\nu}_{1}}}=\frac{2 \mathrm{~K}(m)}{\sqrt{\left(\lambda_{4}-\lambda_{2}\right)\left(\lambda_{3}-\lambda_{1}\right)}} .
$$


The perturbed Whitham equations are given by

$$
\frac{\partial \lambda_{i}}{\partial t}+v_{i} \frac{\partial \lambda_{i}}{\partial x}=-\varepsilon \rho_{i}, \quad i=1,2,3,4
$$

where, as usual,

$$
v_{i}=-\frac{\langle\mathcal{B} / g\rangle}{\langle 1 / g\rangle}=\left(1-\frac{L}{\partial_{i} L} \partial_{i}\right) V, \quad i=1,2,3,4
$$

and

$$
\begin{aligned}
& \rho_{1}=-\frac{\left[\left(2 \lambda_{1}-s / 2\right) \bar{\nu}_{3}+q\right] \mathrm{K}(m)+\left(2 \lambda_{1}-s / 2\right)\left(\bar{\nu}_{3}-\bar{\nu}_{1}\right) \mathrm{E}(m)}{\left(\lambda_{3}-\lambda_{1}\right)\left[\left(\lambda_{4}-\lambda_{1}\right) \mathrm{K}(m)-\left(\lambda_{4}-\lambda_{2}\right) \mathrm{E}(m)\right]}, \\
& \rho_{2}=-\frac{\left[\left(2 \lambda_{2}-s / 2\right) \bar{\nu}_{3}+q\right] \mathrm{K}(m)+\left(2 \lambda_{2}-s / 2\right)\left(\bar{\nu}_{3}-\bar{\nu}_{1}\right) \mathrm{E}(m)}{\left(\lambda_{4}-\lambda_{2}\right)\left[\left(\lambda_{3}-\lambda_{2}\right) \mathrm{K}(m)-\left(\lambda_{3}-\lambda_{1}\right) \mathrm{E}(m)\right]}, \\
& \rho_{3}=-\frac{\left[\left(2 \lambda_{3}-s / 2\right) \bar{\nu}_{3}+q\right] \mathrm{K}(m)+\left(2 \lambda_{3}-s / 2\right)\left(\bar{\nu}_{3}-\bar{\nu}_{1}\right) \mathrm{E}(m)}{\left(\lambda_{3}-\lambda_{1}\right)\left[\left(\lambda_{3}-\lambda_{2}\right) \mathrm{K}(m)-\left(\lambda_{4}-\lambda_{2}\right) \mathrm{E}(m)\right]} \\
& \rho_{4}=-\frac{\left[\left(2 \lambda_{4}-s / 2\right) \bar{\nu}_{3}+q\right] \mathrm{K}(m)+\left(2 \lambda_{4}-s / 2\right)\left(\bar{\nu}_{3}-\bar{\nu}_{1}\right) \mathrm{E}(m)}{\left(\lambda_{4}-\lambda_{2}\right)\left[\left(\lambda_{4}-\lambda_{1}\right) \mathrm{K}(m)-\left(\lambda_{3}-\lambda_{1}\right) \mathrm{E}(m)\right]} .
\end{aligned}
$$

Thus, we see that linear damping in the NLS equation leads to perturbed Whitham equations in simple enough form suitable for applications.

It is easy to check that more general form of nonlinear damping,

$$
\mathrm{i} u_{t}+u_{x x} \pm 2|u|^{2} u=-\mathrm{i} f\left(|u|^{2}\right) u
$$

(with a polynomial function $f(\nu)$ ) as well as action of external potential $V(x)$ slowly depending on $x$,

$$
\mathrm{i} u_{t}+u_{x x} \pm 2|u|^{2} u=V(x) u,
$$

can be treated in the same way and arising in this cases integrals can be reduced to standard elliptic ones, though the formulae become more cumbersome and they will be presented elsewhere together with appropriate application of the Whitham equations.

\section{Conclusion}

The examples considered in the preceding section show that the general formulae (41),(42) provide an effective method of derivation of perturbed Whitham equations. They can be applied to a number of physically important equations integrable by the IST method with $2 \times 2$ matrix or second order scalar spectral problem. For one-phase periodic solutions, the perturbation terms in Whitham equations are expressed in the form of integrals which can be evaluated with the use of known formulae obtained in derivation of the periodic solution under consideration. Thus, the developed here theory permits one to treat effects of perturbations in various physical problems related with modulated nonlinear periodic waves. One such application has been done in the recent paper 31] to evolution of modulated periodic waves in the Bose-Einstein condensate under slow variation of the nonlinear coupling constant. 


\section{Acknowledgments}

I am grateful to F.Kh. Abdullaev and V.V. Konotop for useful discussions and to the staff of Centro de Física da Matéria Condensada, Universidade de Lisboa, for kind hospitality. The work there has been supported by the Senior NATO Fellowship. I thanks also RFBR (Grant 01-01-00696) for partial support.

\section{Appendix. Derivation of the identity (34)}

For simplicity we consider a strictly periodic case when $u_{m}(x, t)$ are periodic functions of $x$ with period $L$. We start from the statement that Eq. (77) is satisfied by the function

$$
\psi^{ \pm}=\sqrt{\tilde{g}} \exp \left( \pm \mathrm{i} \sqrt{-\sigma} \lambda^{r / 2} \int^{x} \frac{\mathrm{d} x}{\tilde{g}}\right)
$$

provided $\tilde{g}$ satisfies Eq. (26) (see, e.g. [32]). These are Baker-Akhiezer functions which can be considered as generalizations of Bloch function solutions of the Schrödinger-like equation (17) with complex "potential" which is a periodic function of $x$ with the period $L$. Then we can introduce formally a "quasi-momentum" $p$ as follows:

$$
\psi^{ \pm}=\exp ( \pm \mathrm{i} p x), \quad p=\frac{\sqrt{-\sigma} \lambda^{r / 2}}{L} \int_{0}^{L} \frac{\mathrm{d} x}{\tilde{g}} .
$$

The "quasi-momentum" $p$ is a functional depending on $u_{m}, m=1, \ldots, N$, and their space derivatives through the coefficients of the expansion (31). If we change $u_{m}$ a little, then $p$ will change a little too, what defines the functional derivative

$$
\frac{\delta p}{\delta u_{m}}=\frac{\sqrt{-\sigma} \lambda^{r / 2}}{L} \frac{\widehat{\delta}}{\delta u_{m}}\left(\frac{1}{\tilde{g}}\right),
$$

where $\widehat{\delta} / \delta u_{m}$ is defined by (351).

On the other hand, this functional derivative can be calculated directly in the following way. We take two functions $u_{m}^{\prime}, u_{m}^{\prime \prime}$ with the same period $L$ and write equations for corresponding Bloch functions,

$$
\psi_{x x}^{\prime}=\mathcal{A}\left(u_{m}^{\prime}\right) \psi^{\prime}, \quad \psi_{x x}^{\prime \prime}=\mathcal{A}\left(u_{m}^{\prime \prime}\right) \psi^{\prime \prime}
$$

which give at once

$$
\left[\psi_{x}^{\prime} \psi^{\prime \prime}-\psi^{\prime} \psi_{x}^{\prime \prime}\right]_{0}^{L}=\int_{0}^{L}\left(\mathcal{A}\left(u_{m}^{\prime}\right)-\mathcal{A}\left(u_{m}^{\prime \prime}\right)\right) \psi^{\prime} \psi^{\prime \prime} \mathrm{d} x
$$

Now, from Eq. A.1.1. we find

$$
\psi_{x}^{ \pm}=\frac{1}{2 \tilde{g}}\left(\tilde{g}_{x} \pm 2 \mathrm{i} \sqrt{-\sigma} \lambda^{r / 2}\right) \psi^{ \pm}
$$


so that if $\psi^{ \pm}$has the property

$$
\psi^{ \pm}(x+L)=\mathrm{e}^{ \pm \mathrm{i} p L} \psi^{ \pm}(x)
$$

then $\psi_{x}^{ \pm}$has the same property,

$$
\psi_{x}^{ \pm}(x+L)=\mathrm{e}^{ \pm \mathrm{i} p L} \psi_{x}^{ \pm}(x)
$$

due to periodicity of $\tilde{g}$. Hence, for $\psi^{\prime}=\psi^{\prime-}$ and $\psi^{\prime \prime}=\psi^{\prime \prime+}$ we find from (A.5) that

$$
\left(\mathrm{e}^{\mathrm{i}\left(p^{\prime \prime}-p^{\prime}\right) L}-1\right)\left(\psi_{x}^{\prime-} \psi^{\prime \prime+}-\psi^{\prime-} \psi_{x}^{\prime \prime+}\right)_{x=0}=\int_{0}^{L}\left(\mathcal{A}\left(u_{m}^{\prime}\right)-\mathcal{A}\left(u_{m}^{\prime \prime}\right)\right) \psi^{\prime-} \psi^{\prime \prime+} \mathrm{d} x .
$$

Taking the difference $u_{m}^{\prime \prime}-u_{m}^{\prime}=\delta u_{m}$ small, we obtain

$$
\psi_{x}^{\prime-} \psi^{\prime \prime+}-\psi^{\prime-} \psi_{x}^{\prime \prime+} \cong-2 \mathrm{i} \sqrt{-\sigma} \lambda^{r / 2}, \quad \mathrm{e}^{\mathrm{i}\left(p^{\prime \prime}-p^{\prime}\right) L}-1 \cong \mathrm{i} \delta p \cdot L,
$$

and for the right hand side of Eq. A.9 we with the same accuracy

$$
-\int_{0}^{L}\left(\mathcal{A}\left(u_{m}^{\prime \prime}\right)-\mathcal{A}\left(u_{m}^{\prime}\right)\right) \tilde{g} d x \cong-\int_{0}^{L}\left[\frac{\partial \mathcal{A}}{\partial u_{m}} \tilde{g}-\frac{\partial}{\partial x}\left(\frac{\partial \mathcal{A}}{\partial u_{m, x}} \tilde{g}\right)+\ldots\right] \delta u_{m} \mathrm{~d} x
$$

so that Eq. (A.9) yields

$$
\frac{\delta p}{\delta u_{m}}=-\frac{1}{2 \sqrt{-\sigma} L \lambda^{r / 2}} \sum_{l=0}^{A_{m}}(-1)^{l} \frac{\partial^{l}}{\partial x^{l}}\left(\frac{\partial \mathcal{A}}{\partial u_{m}^{(l)}} \tilde{g}\right)
$$

where $A_{m}$ is the order of the highest derivative $u_{m}^{\left(A_{m}\right)}$ in $\mathcal{A}$. Then comparison of (A.2) and (A.10) yields the desired identity (34).

\section{References}

[1] Yu.S. Kivshar, B.A. Malomed, Rev. Mod. Phys. 61 (1989) 763.

[2] A.V. Gurevich, L.P. Pitaevskii, ZhETF 65 (1973) 590 [Sov. Phys. JETP 38 (1974) 291]

[3] A.M. Kamchatnov, R.A. Kraenkel, B.A. Umarov, Phys. Rev. E66 (2002) 036609.

[4] A.V. Gurevich, A.L. Krylov, G.A. El, ZhETF 102 (1992) 1524 [Sov. Phys. JETP 75 (1992) 825].

[5] G.A. El, R.H.J. Grimshaw, M.V. Pavlov, Stud. Appl. Math. 106 (2001) 157.

[6] A.V. Ustinov, Physica D 123 (1998) 315.

[7] K.E. Strecker, G.B. Partridge, A.G. Truscott, R.G. Hullet, Nature, 417 (2002) 150.

[8] G.B. Whitham, Proc. Roy. Soc. London, 283 (1965) 238. 
[9] G.B. Whitham, Linear and Nonlinear Waves, Wiley-Interscience, New York, 1974.

[10] H. Flaschka, M.G. Forest, D.W. McLaughlin, Commun. Pure Appl. Math. 33 (1980) 739.

[11] M.G. Forest, D.W. McLaughlin, Stud. Appl. Math. 68 (1983) 11.

[12] M.G. Forest, J.E. Lee, Geometry and modulation theory for periodic nonlinear Schrödinger equation, in: C. Dafermos, J.L. Erickson, D. Kinderlehrer and M. Slemrod, (Eds.), Oscillation Theory, Computation, and Methods of Compensated Compactness, IMA Volumes on Mathematics and its Applications 2, Springer, New York, 1986.

[13] M.V. Pavlov, Teor. Mat. Fiz. 71 (1987) 351 [Theor. Math. Phys. 71 (1987) 584]

[14] A.V. Gurevich, A.L. Krylov, G.A. El, ZhETF 101 (1992) 1797 [Sov. Phys. JETP 74 (1992) 957].

[15] F.R. Tian, Commun. Pure Appl. Math. 46 (1993) 1093.

[16] A.M. Kamchatnov, Phys. Rep. 286 (1997) 199.

[17] M.G. Forest, D.W. McLaughlin, SIAM J. Appl. Math. 44 (1984) 287.

[18] A.V. Gurevich, L.P. Pitaevskii, ZhETF 93 (1987) 871 [Sov. Phys. JETP 66 (1988) 490]

[19] V.V. Avilov, S.P. Novikov, Dokl. Akad. Nauk SSSR, 294 (1987) 325.

[20] S. Myint, R. Grimshaw, Wave Motion, 22 (1995) 215.

[21] E.D. Belokolos, V.Z. Enolskii, A.M. Korostil, Physica D 116 (1998) 253.

[22] M.J. Ablowitz, D.J. Kaup, A.C. Newell, H. Segur, Stud. Appl. Math. 53 (1974) 249.

[23] A.M. Kamchatnov, Nonlinear Periodic Waves and Their Modulations - An Introductory Course, Singapore, World Scientific, 2000.

[24] V.E. Zakharov, A.B. Shabat, ZhETF 61 (1973) 118 [Sov. Phys. JETP 34 (1973) 62].

[25] D.J. Kaup, Progr. Theor. Phys. 64 (1976) 396.

[26] A.M. Kamchatnov, R.A. Kraenkel, J. Phys. A 35 (2002) L13.

[27] V.E. Zakharov, S.V. Manakov, S.P. Novikov, L.P. Pitaevskii, The Theory of Solitons: The Inverse Scattering Method, Moscow, Nauka, 1980; New York, Consultants Bureau, 1984 .

[28] A.M. Kamchatnov, Phys. Lett. A 186 (1994) 387.

[29] S.J. Alber, Complex deformations of integrable Hamiltonians over generalized Jacobi varietes, in: A.S. Fokas, D.J. Kaup, A.C. Newell and V.E. Zakharov, (Eds.), Nonlinear Processes in Physics, Berlin, Springer, 1993, p. 6. 
[30] A.M. Kamchatnov, J. Phys. A 23 (1990) 2945.

[31] F.Kh. Abdullaev, A.M. Kamchatnov, V.V. Konotop, V.A. Brazhnyi, to be published.

[32] A.M. Kamchatnov, J. Phys. A 34 (2001) L441. 